\title{
Profile of everolimus in the treatment of tuberous sclerosis complex: an evidence-based review of its place in therapy
}

This article was published in the following Dove Press journal:

Neuropsychiatric Disease and Treatment

25 August 2016

Number of times this article has been viewed

\author{
Jamie K Capal \\ David Neal Franz \\ Department of Neurology, Cincinnati \\ Children's Hospital Medical Center, \\ Cincinnati, OH, USA
}

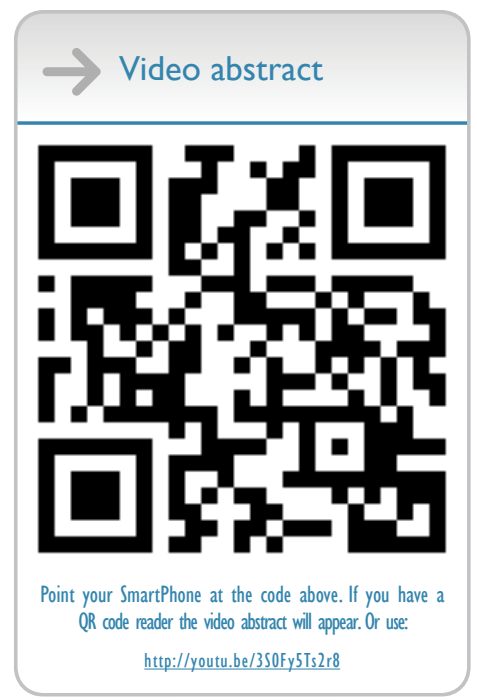

Correspondence: David Neal Franz Department of Neurology, Cincinnati Children's Hospital Medical Center, MLC 20I5, 3333 Burnet Avenue,

Cincinnati, OH 45229, USA

$\mathrm{Tel}+\mathrm{I} 5 \mathrm{I} 36364222$

Fax +15136361888

Email david.franz@cchmc.org
Abstract: Tuberous sclerosis complex (TSC) is a relatively rare genetic disorder, affecting one in 6,000 births. Mammalian target of rapamycin (mTOR) inhibitors, such as everolimus, which have been previously used to prevent solid organ transplant rejection, augment anticancer treatment regimens, and prevent neovascularization of artificial cardiac stents, are now approved for treating TSC-related manifestations, such as subependymal giant cell astrocytomas and renal angiomyolipomas. The use of everolimus in treating subependymal giant cell astrocytomas is supported by long-term Phase II and III clinical trials. Seizures are a common feature in TSC, occurring in up to $96 \%$ of patients. While mTOR inhibitors currently do not have regulatory approval in treating this manifestation, small clinical studies have demonstrated beneficial outcomes with everolimus. Further evidence from a forthcoming Phase III clinical study may provide additional support for the use of everolimus for this indication. Also, there are no approved treatments for TSC-associated neuropsychiatric disorders, which include intellectual disability, behavioral difficulties, and autism spectrum disorder, but preclinical data and small studies have suggested that some neuropsychiatric symptoms may be improved through mTOR inhibition therapy. More evidence is needed, particularly regarding safety in young infants. This review focuses on the current evidence supporting the use of everolimus in neurologic and neuropsychiatric manifestations of TSC, and the place of everolimus in therapy.

Keywords: everolimus, tuberous sclerosis complex, subependymal giant cell astrocytomas, seizures, TAND

\section{Introduction}

Tuberous sclerosis complex (TSC) is a relatively rare disease, with an annual birth incidence estimated to be $\sim 1$ in $6,000 .{ }^{1}$ On a molecular level, TSC results from aberrant hyperactivity of mammalian target of rapamycin (mTOR) that is caused by a mutation in one of two TSC genes, TSC1 and TSC2, which results in an increased cellular proliferation and tumor growth. ${ }^{2}$ TSC affects multiple major organ systems, including the brain, skin, heart, lungs, and kidneys. ${ }^{3}$

Since the discovery of rapamycin in the $1960 \mathrm{~s},{ }^{4}$ mTOR inhibitors, particularly everolimus, have been used to prevent solid organ transplant rejection, augment anticancer treatment regimens, and prevent neovascularization of artificial cardiac stents. ${ }^{5}$ Following the identification of the genes involved in TSC in the $1990 \mathrm{~s},{ }^{6}$ evidence in the 2000s revealed mutations or deletions that allowed mTOR activity to go unchecked (Figure 1) ${ }^{7-9}$ Subsequent clinical trials in patients with TSC led to the approval of the mTOR inhibitor everolimus for TSC-associated subependymal giant cell astrocytoma (SEGA) by US Food and Drug Administration (FDA) in 2010 ${ }^{10}$; it became 


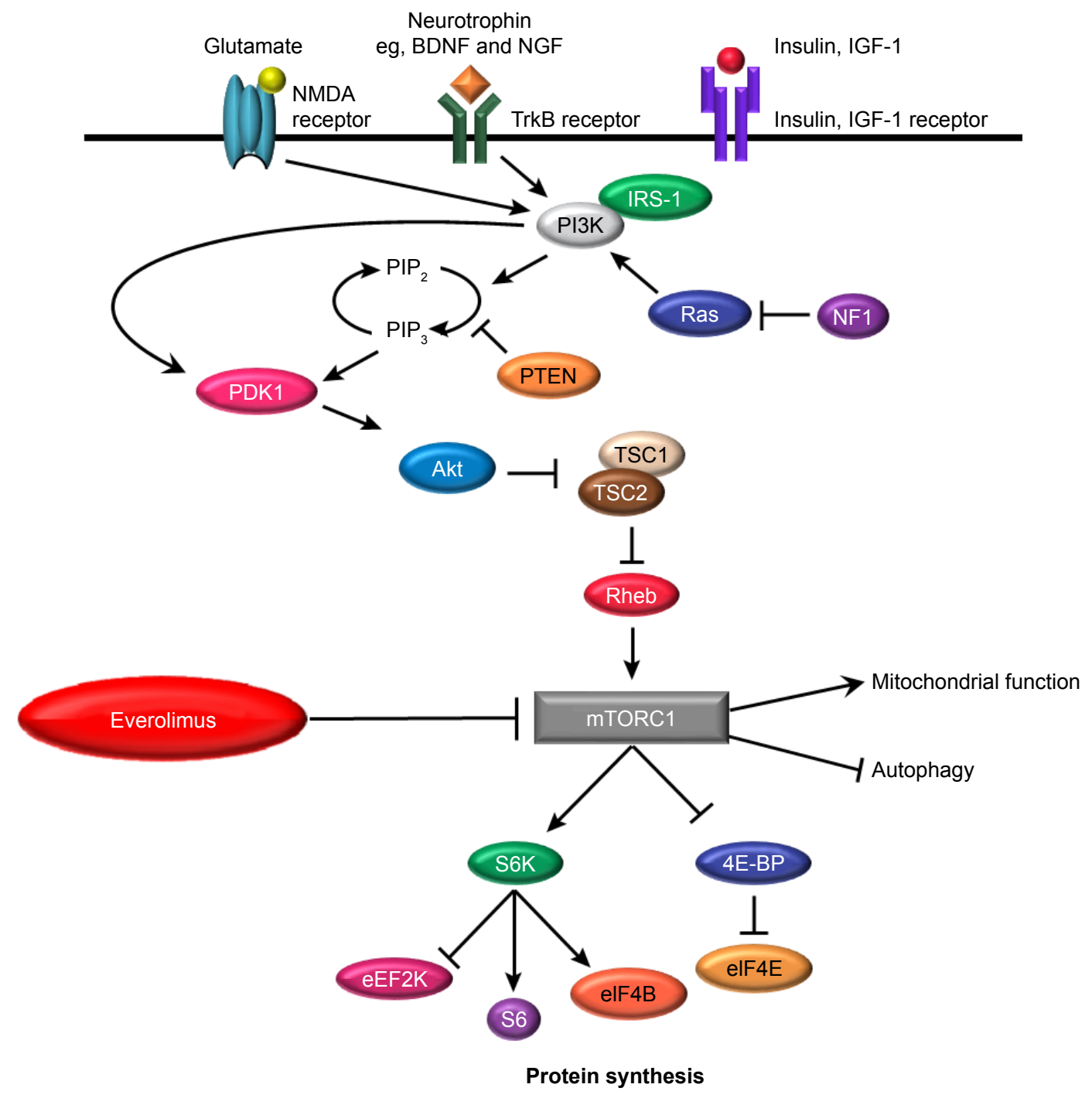

Figure I Everolimus mechanism of action and mTOR pathway.

Note: Adapted by permission from Macmillan Publishers Ltd: Nature Neuroscience. Costa-Mattioli M, Monteggia LM. mTOR complexes in neurodevelopmental and neuropsychiatric disorders. Nat Neurosci. 16(II):1537-1543. Copyright @ 2013. ${ }^{9}$

Abbreviations: NMDA, N-methyl-D-aspartate; BDNF, brain-derived neurotrophic factor; NGF, nerve growth factor; TrkB, tyrosine receptor kinase B; IGF-I, insulin growth factor I; IRS-I, insulin receptor substrate I; PI3K, phosphatidylinositol 3-kinase; PIP, phosphatidylinositol-4,5-bisphosphate; PIP ${ }_{3}$, phosphatidylinositol-3,4,5trisphosphate; NFI, neurofibromin I; PTEN, phosphatase and tensin homolog; PDKI, phosphatidylinositol-dependent kinase I; TSC, tuberous sclerosis complex; Rheb, Ras homolog enriched in brain; mTORCI, mammalian target of rapamycin complex I; S6K, S6 kinase; E-BP, elF4E-binding protein; eEF2K, eukaryotic elongation factor-2 kinase; elF4B, eukaryotic initiation factor $4 \mathrm{~B}$.

the first-ever approved therapy for patients with TSC. Everolimus was also approved by the FDA in 2012 to treat renal angiomyolipomas in patients with TSC. ${ }^{10}$ A summary of the major clinical studies evaluating everolimus in treating TSC is found in Table $1 .{ }^{11-17}$ Although additional definitive, well-designed clinical trials are still needed, mTOR inhibitors have been shown to be beneficial for the treatment of several other clinical manifestations of TSC, including epilepsy, facial angiofibromas, cardiac rhabdomyomas, and lymphangioleiomyomatosis (LAM). ${ }^{17-21}$

This review focuses on the place of everolimus in the treatment of TSC and the evidence supporting its use from a neurologic and neuropsychiatric standpoint.

\section{Subependymal giant cell astrocytomas}

SEGAs are brain tumors composed of glioneuronal cells, usually found near the foramen of Monro. ${ }^{22,23}$ They occur in up to $20 \%$ of patients with TSC and are often present within the first two decades of life..$^{22,23}$ Although SEGAs are benign tumors, due to their capacity for serial growth, they pose a risk for increased intracranial pressure, acute hydrocephalus, and death. ${ }^{23}$

The initial accelerated approval of everolimus for the treatment of growing SEGAs was based on the results of an open-label Phase II study (NCT00411619) in which the effect of everolimus on SEGA growth was examined in 


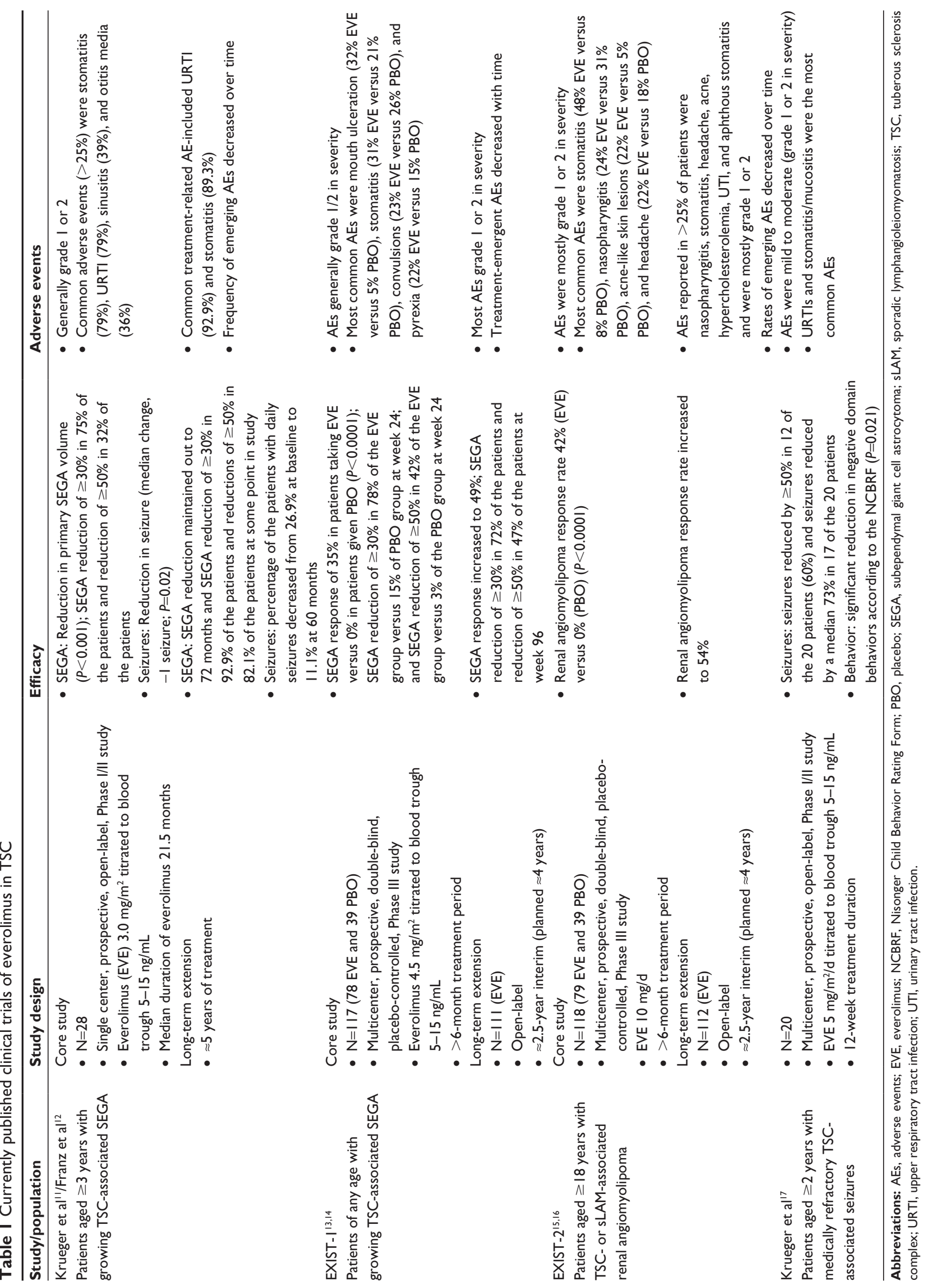


28 patients over a median duration of 21.5 months of treatment. ${ }^{11}$ A clinically significant reduction in tumor size was seen, with reductions of $\geq 30 \%$ in $75 \%$ of the patients and reductions of $\geq 50 \%$ in $32 \%$ of the patients. ${ }^{11}$ In an openlabel extension phase of that trial, patients were allowed to stay on everolimus for up to 5 years. ${ }^{24}$ Twenty-two patients completed the study. Results after 5 years demonstrated that the reduction in SEGA volume was sustained over time. After a median everolimus exposure of 67.8 months, $92.9 \%$ of the patients experienced $\geq 30 \%$ reduction in tumor volume and $82.1 \%$ of the patients experienced $\geq 50 \%$ reduction in tumor volume at some time during the study. ${ }^{12}$ The most common adverse events (AEs) were stomatitis and upper respiratory tract infection (URTI), and no patients discontinued treatment because of an AE. In addition, the frequency of newly emergent AEs decreased over the course of the study. ${ }^{12}$

Following positive results from the open-label Phase II trial, a randomized, double-blind, placebo-controlled, Phase III EXIST-1 study (NCT00789828) was initiated. ${ }^{13}$ One hundred seventeen patients aged 0.8-26.6 years with TSC-associated growing SEGA were randomly assigned in a $2: 1$ ratio to receive everolimus $(n=78)$ and placebo $(n=39)$. After a median follow-up of 9.7 months, $35 \%$ of the patients in the everolimus treatment group achieved a $>50 \%$ reduction in size of SEGA versus $0 \%$ of patients in the placebo group $(P<0.0001) .{ }^{13}$ The study continued with a preplanned open-label extension phase in which any patient remaining in the study could receive everolimus. ${ }^{14}$ An interim analysis examined the 111 patients who received at least one dose of everolimus during a 2- to 3-year period (between August 20, 2009, and January 11, 2013). Median duration of exposure to everolimus was 29.3 months. SEGA response increased from $35 \%$ in the initial trial to $49 \%$ (95\% CI: 39, 58) in the extension phase, and everolimus continued to be safe and well tolerated. ${ }^{14}$ Recently, results of the final analysis of EXIST-1 were presented, and after median treatment duration of 47.1 months, the SEGA response rate increased further to $57.7 \%$ (95\% CI: 47.9, 67.0). Emerging AEs also generally decreased over time. ${ }^{25}$

\section{Seizures}

Seizures are one of the most common features of TSC. Up to $96 \%$ of patients with TSC have seizures, which usually begin within the first 3 years of life and present as focal seizures or infantile spasms. ${ }^{26,27}$ Anticonvulsants are the recommended pharmacologic treatment for TSC-associated epilepsy, particularly vigabatrin for infantile spasms. ${ }^{28,29}$ However, one-third of patients are refractory to treatment, and surgery may be considered. ${ }^{28,29}$ While mTOR inhibitors have not been approved for treating TSC-associated seizures, there is some evidence suggesting that everolimus can improve seizure outcomes.

In the Phase II study discussed earlier that examined the effect of everolimus on SEGA growth, 16 patients had available data from 24-hour electroencephalographic monitoring. Of the eleven patients with a seizure event, nine had a decrease in seizure frequency over the 6-month treatment period. ${ }^{11}$ In the final 5-year analysis of the study, patient-reported seizure frequency decreased from $26.9 \%$ experiencing daily seizures at baseline to $11.1 \%$ at month 60. ${ }^{12}$ Another prospective, open-label, Phase I/II trial evaluated the effect of everolimus in treating refractory epilepsy in patients aged $\geq 2$ years with TSC. ${ }^{17}$ After 12 weeks of treatment with everolimus, parent-reported seizure frequency was reduced by at least $50 \%$ in twelve of the 20 patients, and seizure frequency was reduced by a median of $73 \%$ overall. ${ }^{17}$

Ultimately, the role of everolimus in treating TSCassociated epilepsy is not yet concrete, but EXIST-3, an ongoing, multicenter, Phase III clinical trial evaluating the effect of everolimus on refractory epilepsy associated with TSC (NCT01713946), will help to further answer that question. Results are expected to be available in 2016.

\section{Neuropsychiatric disease}

Neurocognitive disorders, including intellectual disability and behavioral difficulties, affect $\sim 90 \%$ of individuals with TSC. ${ }^{30}$ Furthermore, up to $50 \%$ of those with TSC have autism spectrum disorder (ASD). These neurocognitive and behavioral disorders, combined with psychiatric conditions, together are known as TSC-associated neuropsychiatric disorders (TAND). ${ }^{28}$ The Tuberous Sclerosis Complex Neuropsychiatry Panel met at the 2012 Tuberous Sclerosis Complex International Consensus Conference and defined the term TAND with the goal of providing accurate terminology to the neuropsychiatric diseases seen in TSC, as well as providing guidance on appropriate evaluation and treatment. ${ }^{28,31}$ The Neuropsychiatry Panel recommended that individuals with TSC be screened for TAND at least once per year. The panel devised a TAND checklist, which has since been validated (Table 2). ${ }^{30,31}$

While there are currently no approved medications for the treatment of these symptoms in individuals with TSC, research suggests that mTOR inhibition may provide some benefits. Several preclinical studies have demonstrated that aberrant regulation of mTOR is central to many of the structural, neurophysiologic, and behavioral deficits 
Table 2 Structure of the TAND checklist

\begin{tabular}{ll}
\hline Item & Level of investigation \\
\hline Question I & Basic developmental milestones \\
Question 2 & Current level of functioning \\
Question 3 & Behavioral concerns \\
Question 4 & Psychiatric disorders diagnosed \\
Question 5 & Intellectual ability \\
Question 6 & Academic skills \\
Question 7 & Neuropsychological skills \\
Question 8 & Psychosocial functioning \\
Question 9 & Parent, caregiver, or self-rating \\
& of the impact of TAND \\
Question 10 & Prioritizing list \\
Question II & Additional concerns \\
Question I2 & Health care professional rating \\
& of the impact of TAND \\
\hline
\end{tabular}

Note: Reprinted from Pediatric Neurology,52 /I, De Vries PJ, Tuberous Sclerosis Associated Neuropsychiatric Disorders (TAND) and the TAND Checklist/ 25-35. Copyright 2015 with permission from Elsevier. ${ }^{31}$

Abbreviations: TAND, TSC-associated neuropsychiatric disorders; TSC, tuberous sclerosis complex.

of the disorder. ${ }^{32-40}$ For example, $T s c 1^{+/-}$mice demonstrate impaired learning in hippocampal-dependent learning tasks and abnormal social behavior. ${ }^{37}$ Similarly, $T s c 2^{+/-}$mice show deficits in synaptic plasticity, learning, and memory. ${ }^{36}$ These deficits emerge in the absence of gross structural abnormalities and seizures, demonstrating that other disease mechanisms are involved. ${ }^{36,37}$ The neurocognitive deficits in these same mouse models of TSC can be reversed with treatment with mTOR inhibitors. ${ }^{36}$ Treatment with the mTOR inhibitor rapamycin in adult mice rescued not only synaptic plasticity but also the behavioral deficits in this animal model. ${ }^{36}$ Mouse models demonstrating neurobehavioral deficits, including social impairment and repetitive behaviors, ${ }^{36,39}$ also have shown improvement after treatment with an mTOR inhibitor. ${ }^{39}$ These findings indicate that defects in the Tsc1 or Tsc2 gene result in social impairment typically associated with ASD through dysregulated mTOR signaling and that these impairments may be rescued by pharmacologic mTOR inhibitors, such as rapamycin and everolimus.

Retrospective and small pilot prospective studies have identified specific areas of cognitive impairment and autism spectrum behaviors in early childhood similar to that observed in animal models. ${ }^{41-44}$ A longitudinal cohort study of infants with TSC was performed to define early clinical, behavioral, and biologic markers of ASD. ${ }^{44}$ Infants with TSC and more typically developing controls were recruited beginning as young as 3 months of age and followed longitudinally until they were aged 36 months. At 6 months, infants with TSC demonstrated delays in nonverbal IQ on the Mullen Scales of Early Learning, both in visual receptive and fine motor domains. ${ }^{44}$ Infants with TSC exhibited delays in all developmental domains on the Mullen Scales of Early Learning by the age of 9 months. They also demonstrated more atypical social behaviors as early as 6 months of age in the areas of visual tracking, disengagement of attention, and anticipatory responses, as measured by the Autism Observation Scale for Infants. By the age of 12 months, infants with TSC who were later diagnosed with ASD demonstrated significantly greater cognitive delays compared to infants with TSC without ASD. Developmental trajectories for these groups differed, with a significant decline in nonverbal IQ between 12 months and 36 months in the ASD group only. ${ }^{44}$ Although there was no mention of concomitant use of mTOR inhibitors in this study, it is unlikely that any participants were previously or currently exposed to mTOR inhibitors, and therefore, the impact of mTOR inhibitors on cognitive impairment and ASD cannot be determined.

In an open-label Phase II trial that evaluated the efficacy and safety of rapamycin in adults with renal angiomyolipoma and TSC or LAM, eight patients with TSC had neurocognitive assessments. ${ }^{45}$ Seven of the eight patients tested showed positive change on immediate recall tasks, including list learning, story recall, and figure recall. ${ }^{45}$ These results suggest that mTOR inhibition has the potential to improve certain areas of cognition.

The role of everolimus in treating TAND symptoms in children and adolescents has recently been studied. The RAD001 Neurocognition Trial (NCT01289912) is a Phase II randomized, placebo-controlled trial of everolimus in children and young adults (aged 6-21 years) with TSC. The study was started in 2011 across two sites and evaluated the effect of everolimus on neurocognition over a 6-month period. Neuropsychological measures were given at baseline and 6 months, with additional measures given in the interim at 3 months (www.ClinicalTrials.gov/ct2/ show/NCT01289912). Results of the study have not been published to date. Similarly, the Phase III EXIST-3 study (NCT01713946) is evaluating TAND using the Vineland Adaptive Behavior Scales-II and the Wechsler Non-Verbal Scale of Ability and comparing scores at 18 weeks and every 6 months thereafter with baseline scores (www. ClinicalTrials.gov/ct2/show/NCT01713946). Results from these studies are forthcoming.

\section{Current gaps in knowledge/disease- modifying effects}

Single-gene syndromes with a high prevalence of neurodevelopmental disorders, such as TSC, provide us with 
opportunities to investigate the underlying biology and identify potential treatments. Pharmacologic mTOR inhibitors are proven to be efficacious against multiple TSC disease manifestations. The mTOR inhibitor everolimus is now FDA-approved to treat SEGAs and renal angiomyolipomas in TSC, ${ }^{10}$ but the impact of the treatment on the neurocognitive and neurobehavioral aspects of TSC, particularly in infants and young children, is not yet known nor is the safety of mTOR treatment in this specific population. However, major studies examining these areas, such as EXIST-3, are underway. The severity and underlying causes for neurodevelopmental disorders, such as TAND, are complex and highly variable, which present a major barrier to identification of at-risk infants and development of effective treatments to prevent or alter progression.

All clinical trials with mTOR inhibitors in patients with TSC to date, whether published or in progress, almost exclusively involve or have involved older pediatric and young adult patients. Only EXIST-1, a Phase III clinical trial using everolimus to treat SEGA, reported treating infants under the age of 3 years. ${ }^{13,46}$ Even so, none of the patients treated with everolimus for 6 months were under the age of 1 year. At one of the EXIST-1 sites, four patients were between 1 year and 2 years of age. This center conducted annual neuropsychiatric evaluations outside of the study protocol using the Psyche Cattell test. No significant changes were noted at follow-up compared to baseline. ${ }^{46}$ More detailed assessments to evaluate ASD specifically or other aspects of neurodevelopment were not performed. No study to date has evaluated younger infants treated with mTOR inhibitors using direct, detailed observational assessment tools for objective characterization of TAND deficits. This is a critical age when brain development is at the forefront, and early treatment exposure has the potential to exert dramatic effects on developmental trajectory and overall quality of life in patients with TSC.

Despite optimism for potential benefit of early treatment with mTOR inhibitors in TSC, no studies have provided adequate measure of the safety of mTOR inhibitor treatment in very young infants. mTOR inhibitors have been shown to have immunosuppressive effects, leading to an increased risk of infection in treated individuals. ${ }^{11,24}$ In the prospective, open-label Phase II study using everolimus to treat SEGA, URTIs were reported in $86 \%$ of the patients after a median treatment exposure of 34 months; however, the majority of AEs were either grade 1 or grade 2, and none led to treatment discontinuation. ${ }^{11,24}$ Furthermore, the incidence of infections and other AEs decreased over time. ${ }^{24}$ The youngest enrolled patient was aged 3 years, so no safety data regarding infant use could be generated. An analysis of 18 patients from EXIST-1 aged $<3$ years at everolimus initiation showed that the most common AEs in this population were stomatitis, cough, pharyngitis, and pyrexia. The youngest patient was aged 1.1 years. Serious AEs occurring in two or more patients included pneumonia (three patients), and pyrexia, bronchitis, URTI, and convulsion (two patients each). ${ }^{47}$ Given the prominent role of the mTOR pathway in many normal physiologic functions, there is also the theoretical risk of adverse effects on growth and development, especially early in life. Currently, long-term analyses ( $\approx 5$ years) of patients from EXIST-1 have not demonstrated an impact of everolimus on growth and sexual maturation. ${ }^{48}$ However, it is essential that we establish the basic safety and impact of mTOR inhibitors on neurodevelopment in infants and young children.

An abundance of clinical and basic science evidence suggests that mTOR inhibitors represent a rational candidate for the treatment of TAND. However, mTOR inhibitors have not been adequately evaluated or approved for the treatment of these specific disorders. In a pilot study evaluating everolimus treatment for epilepsy, Krueger et a ${ }^{17}$ utilized two standardized, parental survey instruments (Nisonger Child Behavior Rating Form and Quality of Life for Children with Epilepsy), which included many items overlapping or identifiable as features of TAND. It was noted that several domains and overall quality of life improved after 3 months of treatment. ${ }^{17}$ However, the study was limited by small sample size, unblended treatment, and no direct, observational assessment tools to objectively characterize specific ASD and neurocognitive changes. An earlier prospective, open-label treatment trial using everolimus for SEGA by the same group also attempted to measure neurocognitive function over a 6-month period using direct assessments but was unable to draw any conclusion, as the majority of patients were unable to perform the prescribed assessment battery. ${ }^{11}$

There has been a longstanding interest in identifying treatment strategies for patients with TSC diagnosed at early ages, where the potential impact of mTOR inhibitors or other therapies could drastically improve or even prevent the neurodevelopmental sequelae of ASD and TAND seen in TSC. A prospective, longitudinal study underway at the TSC Clinic at Cincinnati Children's Hospital Medical Center is characterizing safety and neurodevelopment, including autism and neurocognition, in infants with TSC treated with everolimus as clinically indicated. The goals of the study are to determine the effects of everolimus on neurocognitive 
development, including autism, along with its safety profile in this population.

Other existing prospective human clinical trials already in progress are evaluating developmental precursors of ASD in infants with TSC who have never been exposed to mTOR inhibitors (TSC Autism Center of Excellence Network; NCT01780441) and the effect of everolimus on neurocognition in patients aged 6-21 years with TSC (RAD001 study; NCT01289912). While the TSC Autism Center of Excellence Network study provides a well-characterized control group of TSC-related neurodevelopment in infants and toddlers, few were exposed to an mTOR inhibitor, limiting pilot data regarding the safety and treatment effect of mTOR inhibitors in very young individuals. Conversely, the RAD001 Neurocognition Trial is evaluating treatment safety and effect in older children, but potential differences in a younger population that would be targeted for preventative therapies could be missed if they vary significantly due to age, comorbidities, and concurrent treatment, as well as brain maturation and neuroplasticity. Thus, there is a critical need to evaluate the effects of mTOR exposure in young children to fill these gaps and provide a solid and cohesive research strategy for the next logical step - a true early intervention, prevention-based clinical trial using mTOR inhibitors in infants with TSC for the assessment of optimal neurocognitive, developmental, and behavioral outcomes in patients with TSC.

\section{Conclusion}

mTOR inhibitors are already being used to treat several manifestations of TSC, including SEGA and renal angiomyolipomas. However, these medications have the potential to not only treat other manifestations of TSC, such as angiofibromas, cardiac rhabdomyomas, LAM, and epilepsy, but they may also have a role in modifying disease progression at a very young age.

\section{Acknowledgments}

Editorial assistance was provided by Robert Schoen and Traci Stuve of ApotheCom (Yardley, PA, USA). This assistance was funded by Novartis Pharmaceuticals Corporation.

\section{Disclosure}

DNF has received honoraria and travel support from Novartis and Lundbeck Pharmaceuticals. His institution, Cincinnati Children's Hospital Medical Center, has received research support and consulting fees from Novartis. JKC reports no conflicts of interest in this work.

\section{References}

1. Osborne JP, Fryer A, Webb D. Epidemiology of tuberous sclerosis. Ann N Y Acad Sci. 1991;615:125-127.

2. Huang J, Manning BD. The TSC1-TSC2 complex: a molecular switchboard controlling cell growth. Biochem J. 2008;412(2):179-190.

3. Curatolo P, Bombardieri R, Jozwiak S. Tuberous sclerosis. Lancet. 2008;372(9639):657-668.

4. Vézina C, Kudelski A, Sehgal SN. Rapamycin (AY-22,989), a new antifungal antibiotic. I. Taxonomy of the producing streptomycete and isolation of the active principle. $J$ Antibiot (Tokyo). 1975;28(10):721-726.

5. Gabardi S, Baroletti SA. Everolimus: a proliferation signal inhibitor with clinical applications in organ transplantation, oncology, and cardiology. Pharmacotherapy. 2010;30(10):1044-1056.

6. Povey S, Burley MW, Attwood J, et al. Two loci for tuberous sclerosis: one on 9q34 and one on 16p13. Ann Hum Genet. 1994;58(pt 2):107-127.

7. Dabora SL, Jozwiak S, Franz DN, et al. Mutational analysis in a cohort of 224 tuberous sclerosis patients indicates increased severity of TSC2, compared with TSC1, disease in multiple organs. Am J Hum Genet. 2001; 68(1):64-80.

8. Sancak O, Nellist M, Goedbloed M, et al. Mutational analysis of the TSC1 and TSC2 genes in a diagnostic setting: genotype - phenotype correlations and comparison of diagnostic DNA techniques in tuberous sclerosis complex. Eur J Hum Genet. 2005;13(6):731-741.

9. Costa-Mattioli M, Monteggia LM. mTOR complexes in neurodevelopmental and neuropsychiatric disorders. Nat Neurosci. 2013;16(11): $1537-1543$.

10. Novartis Pharma Stein AG. Prescribing Information, Afinitor (Everolimus) Tablets for Oral Administration. Available from: http:// www.pharma.us.novartis.com/product/pi/pdf/afinitor.pdf. Accessed March 8, 2016.

11. Krueger DA, Care MM, Holland K, et al. Everolimus for subependymal giant-cell astrocytomas in tuberous sclerosis. $N$ Engl $J$ Med. 2010;363(19):1801-1811.

12. Franz DN, Agricola K, Mays M, et al. Everolimus for subependymal giant cell astrocytoma: 5-year final analysis. Ann Neurol. 2015;78(6): 929-938.

13. Franz DN, Belousova E, Sparagana S, et al. Efficacy and safety of everolimus for subependymal giant cell astrocytomas associated with tuberous sclerosis complex (EXIST-1): a multicentre, randomised, placebo-controlled phase 3 trial. Lancet. 2013;381(9861):125-132.

14. Franz DN, Belousova E, Sparagana S, et al. Everolimus for subependymal giant cell astrocytoma in patients with tuberous sclerosis complex: 2-year open-label extension of the randomised EXIST-1 study. Lancet Oncol. 2014;15(13):1513-1520.

15. Bissler JJ, Kingswood JC, Radzikowska E, et al. Everolimus for angiomyolipoma associated with tuberous sclerosis complex or sporadic lymphangioleiomyomatosis (EXIST-2): a multicentre, randomised, double-blind, placebo-controlled trial. Lancet. 2013;381(9869):817-824.

16. Bissler JJ, Kingswood JC, Radzikowska E, et al. Everolimus for renal angiomyolipoma in patients with tuberous sclerosis complex or sporadic lymphangioleiomyomatosis: extension of a randomized controlled trial. Nephrol Dial Transplant. 2016;31(1):111-119.

17. Krueger DA, Wilfong AA, Holland-Bouley K, et al. Everolimus treatment of refractory epilepsy in tuberous sclerosis complex. Ann Neurol. 2013;74(5):679-687.

18. Nathan N, Wang J, Li S, et al. Improvement of tuberous sclerosis complex (TSC) skin tumors during long-term treatment with oral sirolimus. J Am Acad Dermatol. 2015;73(5):802-808.

19. Balestri R, Neri I, Patrizi A, Angileri L, Ricci L, Magnano M. Analysis of current data on the use of topical rapamycin in the treatment of facial angiofibromas in tuberous sclerosis complex. $J$ Eur Acad Dermatol Venereol. 2015;29(1):14-20.

20. Tiberio D, Franz DN, Phillips JR. Regression of a cardiac rhabdomyoma in a patient receiving everolimus. Pediatrics. 2011;127(5): e1335-e1337. 
21. McCormack FX, Inoue Y, Moss J, et al; National Institutes of Health Rare Lung Diseases Consortium, MILES Trial Group. Efficacy and safety of sirolimus in lymphangioleiomyomatosis. N Engl J Med. 2011;364(17): 1595-1606.

22. Franz DN, Bissler JJ, McCormack FX. Tuberous sclerosis complex: neurological, renal and pulmonary manifestations. Neuropediatrics. 2010;41(5):199-208.

23. Adriaensen ME, Schaefer-Prokop CM, Stijnen T, Duyndam DA, Zonnenberg BA, Prokop M. Prevalence of subependymal giant cell tumors in patients with tuberous sclerosis and a review of the literature. Eur J Neurol. 2009;16(6):691-696.

24. Krueger DA, Care MM, Agricola K, Tudor C, Mays M, Franz DN. Everolimus long-term safety and efficacy in subependymal giant-cell astrocytoma. Neurology. 2013;80(6):574-580.

25. Franz DN, Belousova E, Sparagana S, et al. Everolimus for subependymal giant cell astrocytoma associated with tuberous sclerosis complex: final 4-year long-term results from EXIST-1 [abstract]. Ann Neurol. 2016;78(suppl 19):S70-S71.

26. Curatolo P, Moavero R, deVries PJ. Neurological and neuropsychiatric aspects of tuberous sclerosis complex. Lancet Neurol. 2015;14(7): 733-745.

27. Curatolo P. Mechanistic target of rapamycin (mTOR) in tuberous sclerosis complex-associated epilepsy. Pediatr Neurol. 2015;52(3):281-289.

28. Krueger DA, Northrup H; International Tuberous Sclerosis Complex Consensus Group. Tuberous Sclerosis complex surveillance and management: recommendations of the 2012 International Tuberous Sclerosis Complex Consensus Conference. Pediatr Neurol. 2013;49(4): 255-265.

29. Krueger DA. Management of CNS-related disease manifestations in patients with tuberous sclerosis complex. Curr Treat Options Neurol.2013; 15(5):618-633.

30. Leclezio L, Jansen A, Whittemore VH, de Vries PJ. Pilot validation of the tuberous sclerosis-associated neuropsychiatric disorders (TAND) checklist. Pediatr Neurol. 2015;52(1):16-24.

31. de Vries PJ, Whittemore VH, Leclezio L, et al. Tuberous sclerosis associated neuropsychiatric disorders (TAND) and the TAND checklist. Pediatr Neurol. 2015;52(1):25-35.

32. Way SW, Rozas NS, Wu HC, et al. The differential effects of prenatal and/or postnatal rapamycin on neurodevelopmental defects and cognition in a neuroglial mouse model of tuberous sclerosis complex. Hum Mol Genet. 2012;21(14):3226-3236.

33. Auerbach BD, Osterweil EK, Bear MF. Mutations causing syndromic autism define an axis of synaptic pathophysiology. Nature. 2011; 480(7375):63-68.

34. Carson RP, Van Nielen DL, Winzenburger PA, Ess KC. Neuronal and glia abnormalities in Tscl-deficient forebrain and partial rescue by rapamycin. Neurobiol Dis. 2012;45(1):369-380.
35. de Vries PJ. Targeted treatments for cognitive and neurodevelopmental disorders in tuberous sclerosis complex. Neurotherapeutics. 2010;7(3): 275-282.

36. Ehninger D, Han S, Shilyansky C, et al. Reversal of learning deficits in a $T s c 2^{+/-}$mouse model of tuberous sclerosis. Nat Med. 2008;14(8): 843-848.

37. Goorden SMI, van Woerden GM, van der Weerd L, Cheadle JP, Elgersma Y. Cognitive deficits in $T s c 1^{+/-}$mice in the absence of cerebral lesions and seizures. Ann Neurol. 2007;62(6):648-655.

38. Hoeffer CA, Tang W, Wong H, et al. Removal of FKBP12 enhances mTOR-raptor interactions, LTP, memory, and perseverative/repetitive behavior. Neuron. 2008;60(5):832-845.

39. Sato A, Kasai S, Kobayashi T, et al. Rapamycin reverses impaired social interaction in mouse models of tuberous sclerosis complex. Nat Commun. 2012;3:1292.

40. Zeng L-H, Ouyang Y, Gazit V, et al. Abnormal glutamate homeostasis and impaired synaptic plasticity and learning in a mouse model of tuberous sclerosis complex. Neurobiol Dis. 2007;28(2):184-196.

41. Jeste SS, Sahin M, Bolton P, Ploubidis GB, Humphrey A. Characterization of autism in young children with tuberous sclerosis complex. J Child Neurol. 2008;23(5):520-525.

42. Humphrey A, Williams J, Pinto E, Bolton PF. A prospective longitudinal study of early cognitive development in tuberous sclerosis: a clinic based study. Eur Child Adolesc Psychiatry. 2004;13(3):159-165.

43. van Eeghen AM, Chu-Shore CJ, Pulsifer MB, Camposano SE, Thiele EA. Cognitive and adaptive development of patients with tuberous sclerosis complex: a retrospective, longitudinal investigation. Epilepsy Behav. 2012;23(1):10-15.

44. Jeste S, Wu JY, Senturk D, et al. Early developmental trajectories associated with ASD in infants with tuberous sclerosis complex. Neurology. 2014;83(2):160-168.

45. Davies DM, de Vries PJ, Johnson SR, et al. Sirolimus therapy for angiomyolipoma in tuberous sclerosis and sporadic lymphangioleiomyomatosis: a phase 2 trial. Clin Cancer Res. 2011;17(12):4071-4081.

46. Kotulska K, Chmielewski D, Borkowska J, et al. Long-term effect of everolimus on epilepsy and growth in children under 3 years of age treated for subependymal giant cell astrocytoma associated with tuberous sclerosis complex. Eur J Paediatr Neurol. 2013;17(5):479-485.

47. Jozwiak S, Kotulska K, Berkowitz N, Brechenmacher T, Franz DN. Safety of everolimus in patients younger than 3 years of age: results from EXIST-1, a randomized, controlled clinical trial. J Pediatr. Epub 2016 Feb 5.

48. Franz DN, Kuperman R, Flamini JR, et al. The effect of everolimus on growth and sexual maturation in patients treated for subependymal giant cell astrocytoma associated with tuberous sclerosis complex: results from the 4-year final analysis of EXIST-1 [abstract]. Ann Neurol. 2015;78(supp1 19):S219.
Neuropsychiatric Disease and Treatment

\section{Publish your work in this journal}

Neuropsychiatric Disease and Treatment is an international, peerreviewed journal of clinical therapeutics and pharmacology focusing on concise rapid reporting of clinical or pre-clinical studies on a range of neuropsychiatric and neurological disorders. This journal is indexed on PubMed Central, the 'PsycINFO' database and CAS,

\section{Dovepress}

and is the official journal of The International Neuropsychiatric Association (INA). The manuscript management system is completely online and includes a very quick and fair peer-review system, which is all easy to use. Visit http://www.dovepress.com/testimonials.php to read real quotes from published authors. 